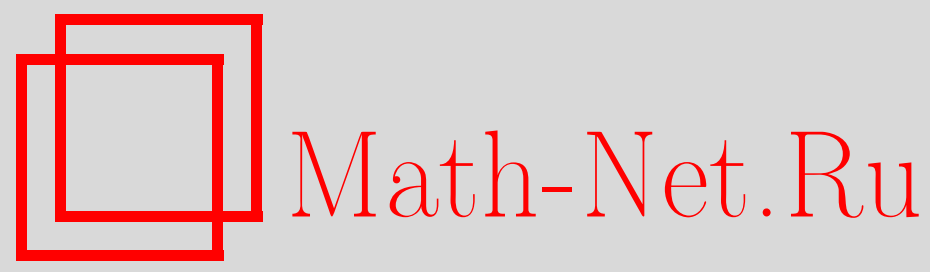

В. М. Климкин, Т. А. Срибная, Равномерная непрерывность семейства слабо регулярных функций множества в топологическом пространстве, Матем. заметки, 2003, том 74, выпуск 1, 60-68

DOI: https://doi.org/10.4213/mzm245

Использование Общероссийского математического портала Math-Net.Ru подразумевает, что вы прочитали и согласны с пользовательским соглашением http://www . mathnet.ru/rus/agreement

Параметры загрузки:

IP : 52.205 .19 .152

26 апреля 2023 г., 13:49:46

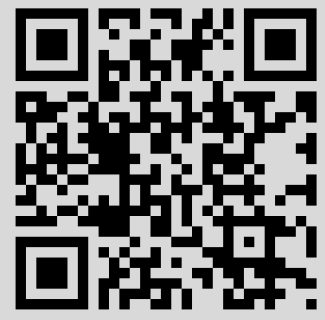




\title{
РАВНОМЕРНАЯ НЕПРЕРЫВНОСТЬ СЕМЕЙСТВА СЛАБО РЕГУЛЯРНЫХ ФУНКЦИЙ МНОЖЕСТВА В ТОПОЛОГИЧЕСКОМ ПРОСТРАНСТВЕ
}

\author{
В. М. Климкин, Т. А. Срибная
}

В работе доказаны условия, при выполнении которых семейство слабо регулярных функций множества, заданных на алгебре $\Sigma, \Sigma \supset \eta$, подмножеств некоторого $\sigma$-топологического пространства $(T, \eta)$ и принимающих значения в произвольном топологическом пространстве, является равномерно непрерывньм.

Библиография: 8 названий.

В работе [1] А. Гротендик доказал, что для равномерной непрерывности семейства ограниченных аддитивных регулярных функций множества, заданных на борелевских множествах локально компактного хаусдорфова топологического пространства, достаточно равномерной непрерывности этого семейства на открытых множествах.

Д. Штейн [2] распространил этот результат на семейство ограниченных аддитивных слабо регулярных функций множества, заданных на борелевских множествах регулярного хаусдорфова топологического пространства.

В данной работе теорема Гротендика доказана для случая, когда функции множества слабо регулярны, неаддитивны, заданы на некоторой алгебре множеств, содержащей класс открытых множеств некоторого $\sigma$-топологического пространства $(T, \eta)$, и принимают значения в произвольном топологическом пространстве. В случае, когда $(T, \eta)$ - хаусдорфово регулярное топологическое пространство, найден ряд условий, эквивалентных условию равномерной непрерьвности семейства $\Phi=\{\varphi\}$ на алгебре $\Sigma$, содержащей класс открытых множеств $\eta$.

Пусть $T$ - некоторое множество, $\eta$ - класс подмножеств множества $T$, замкнутый относительно счетньх объединений и конечных пересечений, $\varnothing \in \eta, T \in \eta$.

Следуя А. Д. Александрову [3], пространство $(T, \eta)$ будем назьвать $\sigma$-топологическим, множества из $\eta$ - открыты.ми, а их дополнения - замкнутыми.

Пусть $\Sigma \supset \eta$ некоторая алгебра подмножеств множества $T ;(X, \tau)$ - произвольное топологическое пространство; $\Phi=\{\varphi\}, \varphi: \Sigma \rightarrow X, \varphi(\varnothing)=e, e \in X,-$ некоторое семейство функций множества; пусть $\mathcal{H}$ - фундаментальная система окрестностей точки $e \in X$.

Для любой функции $\varphi \in \Phi$ и для любого множества $E \in \Sigma$ положим $\widetilde{\varphi}(E)=\{\varphi(F)$, $F \subset E, F \in \Sigma\}$.

Множество $E \in \Sigma$ назовем внешне регулярным $[4$, с. 218] по отношению к семейству функций множества $\Phi=\{\varphi\}$, если для любой окрестности $u \in \mathcal{H}$ существует такое 
открытое множество $U$, что

$$
E \subset U \text { и } \tilde{\varphi}(U \backslash E) \subset u
$$

для любой функции $\varphi \in \Phi$.

Пусть $\mathcal{F}$ - некоторый класс замкнутых множеств. Будем говорить, что функция множества $\varphi \in \Phi$ слабо $\mathcal{F}$-регулярная, если для любого множества $E \in \Sigma$ и для любой окрестности $u \in \mathcal{H}$ существует такое множество $F \in \mathcal{F}, F \subset E$, что $\varphi(E \backslash F) \in u$.

Всюду в дальнейшем последовательность попарно непересекающихся множеств будем назьвать спектром, а убывающую последовательность множеств с пустьм пересечением - локализатором.

Будем говорить, что функции множества семейства $\Phi=\{\varphi\}$ обладают на классе множеств $\mathcal{P} \subset \Sigma$ свойством:

- равномерной исчерпываемости, если для любого спектра $\left\{E_{n}\right\} \subset \mathcal{P}$ соотношение

$$
\lim _{n \rightarrow \infty} \varphi\left(E_{n}\right)=e
$$

выполняется равномерно относительно $\varphi \in \Phi$;

- равномерной слабой непрерывности (и писать $(\Phi, P C H)_{\mathcal{P}}$ ), если для любого локализатора $\left\{E_{n}\right\} \subset \mathcal{P}$ соотношение $(1)$ вьполняется равномерно относительно $\varphi \in \Phi$;

- равномерной непрерывности (и писать $(\Phi, P H)_{\mathcal{P}}$ ), если для любой последовательности $\left\{E_{n}\right\} \subset \mathcal{P}, E_{n} \rightarrow \varnothing$, соотношение (1) вьполняется равномерно относительно $\varphi \in \Phi$

- равномерной квазитреугольности, если для любой окрестности $u \in \mathcal{H}$ существует окрестность $v=v(u), v \in \mathcal{H}$, такая, что для любой функции $\varphi \in \Phi$ и для любых непересекаюшихся множеств $A, B \in \mathcal{P}, A \cup B \in \mathcal{P}$, выполняются условия:

если $\varphi(A) \in v, \varphi(B) \in v$, то $\varphi(A \cup B) \in u$;

если $\varphi(A) \in v, \varphi(A \cup B) \in v$, то $\varphi(B) \in u$.

Если функции множества семейства $\Phi=\{\varphi\}$ равномерно квазитреугольные на $\Sigma$ и $u \in \mathcal{H}$ - некоторая окрестность точки $e \in X$, то всюду в дальнейшем через $\left\{u_{n}\right\} \subset H$ будем обозначать такую последовательность окрестностей, что

$$
u_{0}=u \cap v(u), \quad u_{n}=u_{n-1} \cap v\left(u_{n-1}\right), \quad n=1,2, \ldots .
$$

Примерами семейств функций множества, обладающих свойством равномерной квазитреугольности, являются: семейство $k$-внешних мер [5, с. 913] и, тем более, семейство аддитивных функций множества со значениями в топологической абелевой групе, семейство квазилипшицевых функций множества $[6$, с. 26], семейство квазиадлитивных многозначных соответствий $[7$, с. 38].

Теорема 1. Пусть $(T, \eta)-\sigma$-топологическое пространство, $\Sigma$-некоторая алгебра подмножеств множества $T$, причем $\Sigma \supset \eta ;$ пусть $\mathcal{F}$ - некоторый класс замкнутых множсеств, и пусть $\Phi=\{\varphi\}-$ семейство слабо $\mathcal{F}$-регулярных, равно-

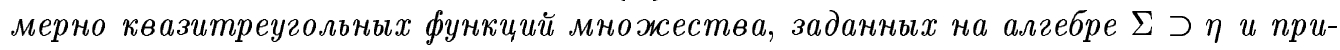
нимающих значения в топологическом пространстве $X$. Если каждое замкнутое множество пространства $(T, \eta)$ является внешне регулярным по отношению $\kappa$ семейству функиий множества $\Phi$, то справедлива импликация

$$
(\Phi, P C H)_{\Sigma} \Rightarrow(\Phi, P H)_{\Sigma} .
$$


ДокАЗАТЕЛЬСТВО теоремы 1 опирается на следуюшие леммы, которые формулируются в предположении, что вьполнены условия теоремы.

ЛЕмма 1. Функиии множества семейства Ф равномерно исчерпывающие на классе множсеств $\mathcal{F}$.

ДоказАТЕЛьСтво. Пусть $\left\{A_{n}\right\}$ - некоторый спектр из $\mathcal{F}$. Так как любое замкнутое множество внешне регулярно по отношению к семейству $\Phi$, существует такое открытое множество $B_{1} \in \eta$, что $A_{1} \subset B_{1}$ и $\widetilde{\varphi}\left(B_{1} \backslash A_{1}\right) \subset u_{1}$ для любой функции $\varphi \in \Phi$.

Положим $C_{2}=A_{2} \backslash B_{1}$. Так как множество $C_{2}$ замкнутое, существует такое открытое множество $B_{2} \in \eta$, что $C_{2} \subset B_{2}$ и $\widetilde{\varphi}\left(B_{2} \backslash C_{2}\right) \subset u_{2}$ для любой функции $\varphi \in \Phi$.

Продолжив процесс, по индукции построим последовательность закнутых множеств $\left\{C_{n}\right\}$ и последовательность открытых множеств $\left\{B_{n}\right\}$ таких, что

$$
\begin{gathered}
C_{1}=A_{1}, \quad C_{n}=A_{n} \backslash \bigcup_{k=1}^{n-1} B_{k}, \quad n=2,3, \ldots, \\
C_{n} \subset B_{n}, \quad \tilde{\varphi}\left(B_{n} \backslash C_{n}\right) \subset u_{n}, \quad n \in \mathbb{N},
\end{gathered}
$$

для любой функции $\varphi \in \Phi$.

Так как

$$
A_{n} \subset C_{n} \cup\left(\bigcup_{k=1}^{n-1}\left(B_{k} \backslash C_{k}\right)\right), \quad n=2,3, \ldots,
$$

каждое множество $A_{n}, n=2,3, \ldots$, можно представить в виде

$$
A_{n}=C_{n} \cup \mathcal{D}_{1} \cup \mathcal{D}_{2} \cup \cdots \cup \mathcal{D}_{n-1},
$$

где $\mathcal{D}_{1}=\left(B_{1} \backslash C_{1}\right) \cap A_{n}$,

$$
\mathcal{D}_{k}=\left(\left(B_{k} \backslash C_{k}\right) \cap A_{n}\right) \backslash \bigcup_{i=1}^{k-1} \mathcal{D}_{i}, \quad k=2,3, \ldots, n-1 .
$$

Из (2) и (4) следует для каждого номера $n=2,3, \ldots$

$$
\widetilde{\varphi}\left(\mathcal{D}_{k}\right) \subset u_{k}, \quad k=1,2, \ldots, n-1,
$$

для любой функции $\varphi \in \Phi$.

Так как множества $\mathcal{D}_{k}, k=1,2, \ldots, n-1$, попарно не пересекаются, из последнего условия в силу свойства равномерной квазитреугольности функций множества семейства $\Phi$ и выбора последовательности $\left\{u_{n}\right\} \subset \mathcal{H}$ следует

$$
\widetilde{\varphi}\left(\bigcup_{k=1}^{n-1} \mathcal{D}_{k}\right) \subset u_{0}, \quad n=2,3, \ldots
$$

для любой функции $\varphi \in \Phi$.

Положим

$$
H_{1}=B_{1}, \quad H_{n}=B_{n} \backslash \bigcup_{k=1}^{n-1} B_{k}, \quad n=2,3, \ldots
$$


Так как

$$
C_{n} \subset H_{n} \quad \text { и } H_{n} \backslash C_{n} \subset B_{n} \backslash C_{n}, \quad n=1,2, \ldots,
$$

учитьвая (2) и свойства последовательности $\left\{u_{n}\right\} \subset \mathcal{H}$, получим при $n=1,2, \ldots$

$$
\widetilde{\varphi}\left(H_{n} \backslash C_{n}\right) \subset u_{1}
$$

для любой функции $\varphi \in \Phi$.

Положим

$$
F_{n}=\bigcup_{k=n}^{\infty} H_{k}, \quad n=1,2, \ldots
$$

Очевидно, что

$$
F_{n}=H_{n} \cup F_{n+1} \quad \text { и } H_{n} \cap F_{n+1}=\varnothing, \quad n=1,2, \ldots
$$

Так как функции множества семейства $\Phi=\{\varphi\}$ обладают свойством $P C H$ на $\Sigma$, а последовательность множеств $F_{n}, n=1,2, \ldots$, образует локализатор из $\Sigma$, сушествует такой номер $n_{0}$, что для всех номеров $n>n_{0}$ справедливо

$$
\varphi\left(F_{n}\right) \in u_{2}
$$

для любой функции $\varphi \in \Phi$.

Так как функции множества семейства $\Phi$ равномерно квазитреугольные, из (7) и (8) следует, что для всех $n>n_{0}$

$$
\varphi\left(H_{n}\right) \in u_{1}
$$

для любой функции $\varphi \in \Phi$.

Аналогично, из (6), (9) и соотношения

$$
H_{n}=\left(H_{n} \backslash C_{n}\right) \cup C_{n}, \quad n=1,2, \ldots,
$$

получим, что для всех номеров $n>n_{0}$ справедливо

$$
\varphi\left(C_{n}\right) \in u_{0}
$$

для любой функции $\varphi \in \Phi$. Отсюда в силу $(3),(5)$ и выбора окрестности $u_{0} \in \mathcal{H}$ получим, что для всех номеров $n>n_{0}$

$$
\varphi\left(A_{n}\right) \in u
$$

для любой функции $\varphi \in \Phi$. Лемма 1 доказана.

ЛЕмма 2. Функиии множества семейства Ф равномерно исчерпывающие на алгебре $\Sigma$. 
ДокАЗАТЕЛЬСТво. Предположим противное. Тогда существуют спектр $\left\{E_{n}\right\} \subset \Sigma$, окрестность $u \in \mathcal{H}$ и последовательность функций $\left\{\varphi_{n}\right\} \subset \Phi$, для которых

$$
\varphi_{n}\left(E_{n}\right) \notin u, \quad n=1,2, \ldots .
$$

Так как каждая функция $\varphi_{n}$ слабо $\mathcal{F}$-регулярная, существуют множества $F_{n} \in \mathcal{F}$ такие, что

$$
F_{n} \subset E_{n} \quad \text { и } \varphi_{n}\left(E_{n} \backslash F_{n}\right) \in u_{0}, \quad n=1,2, \ldots
$$

По лемме 1 существует номер $n_{0}$ такой, что

$$
\varphi_{n}\left(F_{n}\right) \in u_{0} \quad \text { при всех } n>n_{0} .
$$

Так как

$$
E_{n}=F_{n} \cup\left(E_{n} \backslash F_{n}\right),
$$

TO

$$
\varphi_{n}\left(E_{n}\right) \in u, \quad n>n_{0} .
$$

Получили противоречие. Лемма 2 доказана.

ЛЕмма 3. Для любой окрестности $u \in \mathcal{H}$ и для любого локализатора $\left\{E_{n}\right\} \subset \Sigma$ существует номер $n_{0}$ такой, что для любого номера $n>n_{0}$ соотношение

$$
\widetilde{\varphi}\left(E_{n}\right) \subset u
$$

выполняется для любой функиии $\varphi \in \Phi$.

ДокАЗАТЕЛЬСТво. Предположим противное. Тогда существуют локализатор $\left\{E_{n}\right\} \subset \Sigma$, окрестность $u \in \mathcal{H}$ и последовательность функций $\left\{\varphi_{n}\right\} \subset \Phi$ такие, что

$$
\widetilde{\varphi}_{n}\left(E_{n}\right) \notin u, \quad n=1,2, \ldots .
$$

Отсюда следует, что существует множество $A_{1} \in \Sigma$ такое, что

$$
A_{1} \subset E_{1}, \quad \varphi_{1}\left(A_{1}\right) \notin u \text {. }
$$

Так как $\left\{A_{1} E_{n}\right\}$ - локализатор из $\Sigma$, а функции множества семейства $\Phi$ обладают свойством $P C H$ на $\Sigma$, существует номер $n_{1}$ такой, что

$$
\varphi_{1}\left(A_{1} E_{n_{1}}\right) \in u_{0} .
$$

Отсюда в силу (10) и выбора окрестности $u_{0} \in \mathcal{H}$ получаем

$$
\varphi_{1}\left(A_{1} \backslash\left(A_{1} E_{n_{1}}\right)\right) \notin u_{0} .
$$

Аналогично, найдем множество $A_{n_{1}} \in \Sigma$ и номер $n_{2}>n_{0}$ такие, что

$$
A_{n_{1}} \subset E_{n_{1}}, \quad \varphi_{n_{1}}\left(A_{n_{1}}\right) \notin u, \quad \varphi_{n_{1}}\left(A_{n_{1}} E_{n_{2}}\right) \in u_{0} .
$$

Тогда

$$
\varphi_{n_{1}}\left(A_{n_{1}} \backslash A_{n_{1}} E_{n_{2}}\right) \notin u_{0} .
$$

Продолжив процесс, по индукции построим подпоследовательность номеров $\left\{n_{k}\right\}$ и последовательности множеств $\left\{A_{n_{k}}\right\}$ и $\left\{E_{n_{k}}\right\}$ из $\Sigma$ такие, что $A_{n_{k}} \subset E_{n_{k}}$ и

$$
\varphi_{n_{k}}\left(A_{n_{k}} \backslash A_{n_{k}} E_{n_{k+1}}\right) \notin u_{0} .
$$

Так как множества $\left\{A_{n_{k}} \backslash A_{n_{k}} E_{n_{k+1}}\right\}$ образуют спектр из $\Sigma$, последнее соотношение противоречит свойству равномерной исчерпьваемости на $\Sigma$ функций множества семейства $\Phi$. Лемма 3 доказана. 
ДОКАЗАТЕЛЬСТВо ТЕОРЕМЫ 1. Предположим, что фУнкции множества семейства $\Phi$ не обладают свойством $(P H)_{\Sigma}$. Тогда существуют последовательность $\left\{E_{n}\right\} \subset \Sigma$, $E_{n} \rightarrow \varnothing$, окрестность $u \in \mathcal{H}$ и последовательность $\left\{\varphi_{n}\right\} \subset \Phi$ такие, что

$$
\widetilde{\varphi}_{n}\left(E_{n}\right) \notin u .
$$

Так как функции множества семейства $\Phi$ равномерно исчерпьвающие на $\Sigma$, существует номер $n_{1}$ такой, что для всех номеров $n>n_{1}$ и для всех функций множества $\varphi \in \Phi$ справедливо

$$
\widetilde{\varphi}\left(E_{n} \backslash \bigcup_{k=1}^{n}\left(E_{k}\right)\right) \subset u_{1} .
$$

Положим

$$
B_{1}=\bigcup_{k=1}^{n_{1}} E_{k} .
$$

Аналогично, для последовательности $\left\{E_{n} B_{1}\right\}_{n>n_{1}}$ найдем номер $n_{2}$ такой, что для всех $n>n_{2}$ и для всех функций $\varphi \in \Phi$ справедиво

$$
\widetilde{\varphi}\left(E_{n} B_{1} \backslash B_{2}\right) \subset u_{2}, \quad \text { где } B_{2}=\bigcup_{k=n_{1}+1}^{n_{2}} E_{k} B_{1} .
$$

Продолжив процесс, по индукции построим последовательность номеров $\left\{n_{k}\right\}$ и локализатор $\left\{B_{k}\right\} \subset \Sigma$ такие, что

$$
B_{0}=T, \quad B_{k}=\bigcup_{i=n_{k-1}+1}^{n_{k}} E_{i} B_{k-1}, \quad k=1,2, \ldots, \quad \widetilde{\varphi}\left(E_{n} B_{k-1} \backslash B_{k}\right) \subset u_{k}
$$

для всех $n>n_{k}$ и для всех функций $\varphi \in \Phi$.

Отсюда в силу построения последовательности $\left\{u_{k}\right\} \subset \mathcal{H}$ получим

$$
\widetilde{\varphi}\left(\bigcup_{i=1}^{k}\left(E_{n} B_{i-1} \backslash B_{i}\right)\right) \subset u_{0}
$$

для любого номера $k=1,2, \ldots$ и для любой функции $\varphi \in \Phi$.

Так как для любых номеров $k$ и $n>n_{k}$ справедливо соотношение

$$
E_{n} \subset\left(\bigcup_{i=1}^{k}\left(E_{n} B_{i-1}\right)\right) \cup B_{k},
$$

из (11) и (12) следует, что при $n>n_{k}, k=1,2, \ldots$,

$$
\widetilde{\varphi}_{n}\left(B_{k}\right) \notin u_{0},
$$

что противоречит лемме 3 . Теорема доказана. 
ОПРЕДЕЛЕНИЕ. Пусть $\mathcal{L}$ - класс всех замкнутых множеств $\sigma$-топологического пространства $(T, \eta), \mathcal{F} \subset \mathcal{L}$. Будем говорить, что классы множеств $\mathcal{F}$ и $\mathcal{L} \eta$-отделимы, если для любых непересекающихся множеств $F \in \mathcal{F}$ и $L \in \mathcal{L}$ существуют такие открытые непересекающие множества $U, V \in \eta$, что $F \subset U, L \subset V$.

ПримеРЫ. 1. Пусть $(T, \eta)$ - регулярное топологическое пространство; пусть $\mathcal{L}-$ класс всех замкнутых множеств пространства $(T, \eta)$. Если $\mathcal{F}$ - некоторый класс замкнутых компактных множеств пространства $(T, \eta)$, то классы множеств $\mathcal{F}$ и $\mathcal{L} \eta$-отделимы.

2. Пусть $(T, \eta)$ - регулярное хаусдорфово топологическое пространство; пусть $\mathcal{L}$ класс всех замкнутых множеств пространства $(T, \eta)$. Если $\mathcal{F}$ - некоторьй класс компактных множеств пространства $(T, \eta)$, то классы множеств $\mathcal{F}$ и $\mathcal{L} \eta$-отделимы.

3. Пусть $(T, \eta)$ - нормальное топологическое пространство; пусть $\mathcal{L}$ - класс всех замкнутых множеств пространства $(T, \eta)$. Если $\mathcal{F}$ - некоторьй класс замкнутых множеств пространства $(T, \eta)$, то классы множеств $\mathcal{F}$ и $\mathcal{L} \eta$-отделимы.

СлеДствИЕ. Пусть $(T, \eta), \quad \Sigma u \mathcal{F}$ mе жее, что и в теореме 1 . Пусть $\mathcal{L}-$ класс всех замкнутых мнохсеств пространства $(T, \eta)$ и классы множеств $\mathcal{F} u$ $\mathcal{L} \eta$-отделимы. Если $\Phi=\{\varphi\}$ - семейство слабо $\mathcal{F}$-регулярных, равномерно квазитреугольных функиий множества, заданных на алгебре $\Sigma \supset \eta$ и принимающих значения в топологическом пространстве $X$, то справедлива импликачия

$$
(\Phi, P C H)_{\Sigma} \Rightarrow(\Phi, P H)_{\Sigma} .
$$

ДокАЗАТЕЛЬСТво. Если классы множеств $\mathcal{F}$ и $\mathcal{L} \eta$-отделимы, то каждоемножество $L \in \mathcal{L}$ является внешне регулярным по отношению к семейству функций множества $\Phi=$ $\{\varphi\}$. Осталось применить теорему 1.

Теорема 2. Пусть $(T, \eta)$ - регулярное хаусдорфово топологическое простран-

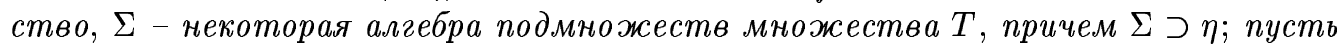
$\mathcal{F}$ - класс компактных множеств пространства $(T, \eta), a \Phi=\{\varphi\}-$ семейство слабо $\mathcal{F}$-регулярных, равномерно квазитреугольных функиий мнохества, заданных на алгебре $\Sigma \supset \eta$ и принимающих значения в топологическом пространстве $X$.

Следующие условия әквивалентны.

I. Функиии множества семейства Ф равномерно исчерпывающие на классе $\eta$ открытых множеств.

II. Для любого множества $E \in \Sigma$ и для любой окрестности $u \in \mathcal{H}$ существует такое компактное мнохсество $F$, что $F \subset E$ u $\widetilde{\varphi}(E \backslash F) \subset u$ для любой функиии $\varphi \in \Phi$.

III. Функиии множества семейства Ф равномерно непрерывные на $\Sigma$.

IV. Функиии множества семейства Ф равномерно слабо непрерывные на классе $\eta$ открытых множеств.

ДокАЗАТЕЛьство. 1. Если функции множества семейства $\Phi$ равномерно исчерпываюшие на классе $\eta$ открытых множеств, то по лемме 3 работы [8, с. 106-107] для любого множества $E \in \Sigma$ и для любой окрестности $u \in \mathcal{H}$ существует такой набор попарно непересекающихся компактных множеств $F_{1}, \ldots, F_{n}$, что

$$
F_{i} \subset E, \quad i=1, \ldots, n, \quad \widetilde{\varphi}\left(E \backslash \bigcup_{i=1}^{n} F_{i}\right) \subset u
$$


для любой функции $\varphi \in \Phi$.

Отсюда следует справедливость импликации (I) $\Rightarrow(\mathrm{II})$.

2. Пусть выполнено условие (II). Покажем, что в этом случае вьполняются условия теоремы 1.

Пусть $u \in \mathcal{H}$, а $L$ - некоторое замкнутое множество. По условию существует такое компактное множество $F$, что

$$
F \subset T \backslash L \text { и } \tilde{\varphi}((T \backslash L) \backslash F) \subset u
$$

для любой функции $\varphi \in \Phi$. Положим $U=T \backslash F$; тогда

$$
L \subset U \text { и } \tilde{\varphi}(U \backslash L) \subset u
$$

для любой функции $\varphi \in \Phi$.

Пусть $\left\{E_{n}\right\}$ - некоторый локализатор из $\Sigma$. По условию для каждого множества $E_{n}$ существует такое компактное множество $F_{n}$, что

$$
F_{n} \subset E_{n}, \quad \widetilde{\varphi}\left(E_{n} \backslash F_{n}\right) \subset u_{n}
$$

для любой функции $\varphi \in \Phi$.

Так как

$$
\bigcap_{n=1}^{\infty} F_{n}=\varnothing
$$

существует номер $n_{0}$ такой, что

$$
\bigcap_{n=1}^{n_{0}} F_{n}=\varnothing
$$

Из соотношений

$$
E_{n_{0}}=E_{n_{0}} \backslash \bigcap_{n=1}^{n_{0}} F_{n}=\bigcup_{n=1}^{n_{0}}\left(E_{n_{0}} \backslash F_{n}\right) \subset \bigcup_{n=1}^{n_{0}}\left(E_{n} \backslash F_{n}\right)
$$

в силу условия (13) и выбора последовательности $\left\{u_{n}\right\} \subset \mathcal{H}$ следует

$$
\widetilde{\varphi}\left(E_{n_{0}}\right) \subset u
$$

для любой функции $\varphi \in \Phi$.

Таким образом, условия теоремы 1 вьполнены. Следовательно, функции множества семейства $\Phi$ равномерно непрерывные на $\Sigma$ и импликация (II) $\Rightarrow$ (III) доказана.

3. Справедливость импликации (III) $\Rightarrow(\mathrm{IV})$ следует из определений свойства равномерной непрерывности, свойства равномерной слабой непрерывности и условия $\Sigma \supset \eta$.

4. Пусть функции множества семейства $\Phi$ равномерно слабо непрерывные на классе $\eta$ открытых множеств. Пусть $u \in \mathcal{H} ;\left\{U_{n}\right\}$ - некоторьй спектр из $\eta$. Положим

$$
E_{n}=\bigcup_{k=n}^{\infty} U_{k}, \quad n=1,2, \ldots
$$


Так как последовательность множеств $\left\{E_{n}\right\}$ является локализатором из $\eta$, существует номер $n_{0}$ такой, что для всех номеров $n>n_{0}$ и для любой функции $\varphi \in \Phi$ справедливо

$$
\varphi\left(E_{n}\right) \in u_{0}
$$

Отсюда и из соотношения $E_{n}=U_{n} \bigcup E_{n+1}$ в силу выбора окрестности $u_{0} \in \mathcal{H}$ следует, что для любого номера $n>n_{0}$ и для любой функции $\varphi \in \Phi$ справедливо

$$
\varphi\left(U_{n}\right) \in u
$$

что означает равномерную исчерпываемость функций множества семейства $\Phi$ на классе $\eta$ открытых множеств. Теорема доказана.

\section{СПИСОК ЦИТИРОВАННОЙ ЛИТЕРАТУРЫ}

[1] Grothendieck A. Sur les applications linéaires faiblement compactes d'espaces du type $C(K)$ // Canad. J. Math. 1953. V. 51. P. 129-173.

[2] Stein J. D. Uniform absolute continuity in spaces of set functions // Proc. Amer. Math. Soc. 1975. V. 51. № 1. P. 137-140.

[3] Александров А. Д. Аддитивные функции множества в абстрактных пространствах // Матем. сб. 1941. Т. 9(51). С. 563-628.

[4] Халмош П. Теория меры. М.: ИЛ, 1953.

[5] Саженков А. Н. Ограниченность векторных мер // Матем. заметки. 1979. Т. 25. №6. C. $913-917$.

[6] Гусельников Н. С. О продолжении квазилипшицевых функций множества // Матем. заметки. 1975. Т. 17. №1. С. 21-31.

[7] Drewnowski L. Additive and countable additive correspondences // Rocz. Pol. Tow. Mat. Ser. I. 1976. V. 19. № 1. P. 25-54.

[8] Климкин В. М., Срибная Т. А. Сходимость последовательности слабо регулярных функций множества // Матем. заметки. 1997. Т. 62. №1. С. 103-110. 